\title{
Psychological Stress Induces an Increase in Cholinergic Enteric Neuromuscular Pathways Mediated by Glucocorticoid Receptors
}

Justine Blin

Nantes Université, CHU Nantes, INSERM, IMAD

Camille Gautier

Nantes Université, CHU Nantes, INSERM, IMAD

Philippe Aubert

Nantes Université, CHU Nantes, INSERM, IMAD

Tony Durand

Nantes Université, CHU Nantes, INSERM, IMAD

Thibauld Oullier

Nantes Université, CHU Nantes, INSERM, IMAD

Philippe Naveilhan

Nantes Université, CHU Nantes, INSERM, IMAD

Damien Masson

Nantes Université, CHU Nantes, INSERM, IMAD

Michel Neunlist

Nantes Université, CHU Nantes, INSERM, IMAD

Kalyane Bach-Ngohou ( $\sim$ Kalyane.bach@chu-nantes.fr)

Nantes Université, CHU Nantes, INSERM, IMAD

\section{Research Article}

Keywords: Brain-gut axis, stress, enteric nervous system, HPA, Glucocorticoid receptor, GR, NR3C1, corticosterone, ChAT, water avoidance stress, CORT108297

Posted Date: January 18th, 2022

DOI: https://doi.org/10.21203/rs.3.rs-1229272/v1

License: (c) (1) This work is licensed under a Creative Commons Attribution 4.0 International License.

Read Full License 


\section{Abstract}

Repeated acute stress (RASt) is known to be associated with gastrointestinal dysfunctions. However, mechanisms underlying these effects are not fully understood. While glucocorticoids are clearly identified as hormones of stress, their implication, and those of their specific glucocorticoid receptors (GR), in RASt induced gut dysfunctions remain unclear. The aim of our study was to evaluate the involvement of GR on RASt induced changes in gut motility, particularly through the enteric nervous system (ENS). Using a murine water avoidance stress model, we characterized the impact of RASt upon ENS phenotype and colonic motility. Next, the expression of GR onto the ENS and their functional impact upon RASt induced changes in ENS phenotype and motor response were evaluated. We showed that the GR was expressed in myenteric neurons in the distal colon under basal condition, and that RASt enhanced its nuclear translocation. RASt increased the proportion of ChAT-IR neurons, acetylcholine tissue concentration, and enhanced cholinergic neuromuscular transmission as compared to control. Finally, we showed that GR specific antagonist (CORT108297) prevented the increase of acetylcholine and in vivo colonic motility. Altogether our study suggests that RASt-induced functional changes in motility are, at least partly, due to GR dependent enhanced cholinergic ENS phenotype.

\section{Introduction}

Repeated acute stress (RASt) is known to be associated with gastrointestinal (GI) disorders or dysfunctions such as altered gastrointestinal motility, leading to decreased gastric emptying and enhanced colonic transit time, or altered epithelial functions such as increased epithelial secretion or permeability (1-3). However, mechanisms underlying RASt induced changes in gut functions, in particular mediators and receptors involved, have not yet been fully decrypted $(1,2,4)$.

RASt is associated with stimulation of hypothalamic-pituitary-adrenal (HPA) axis. Activation of HPA axis is characterized by secretion of corticotropin releasing hormone (CRH) by hypothalamus, which induces release of ACTH by pituitary gland, and finally secretion of glucocorticoids (GC) by adrenal glands (5-8). Among mediators released following HPA activation and involved in RASt effects upon GI functions, CRH has been the most widely studied. In particular, $\mathrm{CRH}$ modulates $\mathrm{Gl}$ functions via neuronal pathways involved in the central nervous system (CNS) $(9,10)$ and via the autonomic nervous system such as the sympathetic nervous system or the enteric nervous system (ENS) $(5-8,11)$.

In particular, $\mathrm{CRH}$ can directly act on neurons of the ENS, an integrative neuronal network located all along the gastrointestinal tract, involved in the regulation of gastrointestinal functions such as motility and secretion (12-14). CRH effects upon the ENS were shown to be mediated in a region and function dependent manner by activation of two receptors sub-types, CRF-R1 and CRF-R2. Numerous studies have tested the hypothesis that stress could impact digestive function by acting on these receptors (15). Among them, CRF-R1 is expressed in a region-specific manner, mainly in the ileum and colon of rodents and humans. Activation of CRF-R1 by CRH induces a colonic motor response similar to the stress-induced 
one. Conversely, the activation of CRF-R2 receptors increases gastric transit time (9). In addition, activation of CRF-R2 by CRH increases mucosal chloride secretion in the colon via enteric pathways (16).

Besides $\mathrm{CRH}$, the role of glucocorticoids (GC), cortisol in human or corticosterone in murine, in RASt mediated effects upon GI functions, and in particular its putative impact upon the ENS remains largely unknown. GC effects are mediated via two major nuclear receptors, i.e. MR and GR. GC have a high affinity for MR receptors, which are often activated under basal conditions. In contrast, GC have a low affinity for GR receptors which are activated under 'inducible' condition, such as following HPA activation (17-19). GC activation of GR results in nuclear translocation of cytoplasmic GR and binding to GR response element, leading to various transcriptional process. Effects of corticosterone are limited by its degrading enzymes $11 \beta H S D-2$ (20). Concerning the ENS, although a recent study identified $11 \beta H S D-2$ in enteric neurons, the expression of GR and its putative involvement in RASt effects upon GI functions is currently unknown.

GR expression has however been identified in other neuronal system such as the sympathetic system such as dorsal root sensory neurons or in specific brain structure (21). In neurons, activation of GR by GC has been shown to regulate neuromediator expression or different neuronal process and plasticity such as sprouting $(17,21,22)$. In the ENS, corticosterone has been shown to increase the proportion of choline acetyltransferase (ChAT) in primary ENS culture (23). In addition, corticosterone has been shown to modulate Ca concentration in enteric neurons (24). However, the involvement of GR in GC mediated effects upon the ENS remains largely unknown.

In this context, our study aimed at determining whether the ENS express GR receptors and whether RAStinduced effects on ENS phenotype and on functional remodelling are mediated, at least in part, via GR dependent pathways.

\section{Material And Methods}

\section{Animals}

Our study was carried out in accordance with French standard ethical guidelines for laboratory animals (Agreement 02376.01) and with ARRIVE guidelines (https://arriveguidelines.org). All experiments involving mice were approved by the ethics committee for animal experimentation of Pays de la Loire (Apafis 6751) as Nantes University CHU, of which our INSERM unit is part, is geographically located in the Pays de la Loire. Adult male C57BL/ 6 mice (6-7 weeks; Janvier Labs, Le Genest-Saint-Isle, France) weighing $25-30 \mathrm{~g}$ were used. Mice were housed in cages with free access to food and tap water. Animals were quarantined under controlled conditions illumination ( $12 \mathrm{~h} \mathrm{light/dark} \mathrm{cycle),} \mathrm{temperature,} \mathrm{and}$ humidity.

\section{Water avoidance stress (WAS) protocol}


For stress exposure, a single $1 \mathrm{~h}$ WAS in between 8 AM to 9 AM in the morning, was performed daily at PD35 for 4 consecutive days, corresponding to the repeated acute stress protocol. Mice were placed on an elevated circular platform ( $10 \mathrm{~cm}$ height ; $4 \mathrm{~cm}$ diameter), positioned at the center of a plastic tank (52 $x 37 \times 27 \mathrm{~cm}^{3}$ ) filled with water at room temperature up to $1 \mathrm{~cm}$ of the top of the platform adapted from previous study $(3,25)$.

\section{Pharmacological treatment protocol}

The specific GR antagonist, CORT108297 (kindly provided by Dr Azel Hunt, Corcept, Drive Menlo Park, CA, USA) or the vehicle (sesame oil, Millipore Sigma, Burlington, MA, USA) were injected intraperitoneally at the dose of $85 \mathrm{mg} / \mathrm{kg}$ (26) 1 hour before the WAS procedure.

\section{In vivo measurement of colonic motility}

As previously described $(27,28)$, to assess the fecal pellet output (FPO), control mice were placed individually in clean cages without bedding, food, or water for fecal pellet collection during $1 \mathrm{~h}$. For mice subjected to WAS, the fecal pellet collection was performed for $1 \mathrm{~h}$ during the WAS time period. Fecal pellets were collected and counted immediately after expulsion.

\section{Ex vivo measurement of colonic motility}

As previously described $(27,29)$, at the end of the fecal pellet collection for the control mice and after the WAS treatment the fourth day, animals were killed by cervical dislocation. Tissues were immediately transferred in cold HBSS (Eurobio, Courtaboeuf, France) after surgical resection and brought to the laboratory. Strips of longitudinal muscle were dissected and placed in organ chamber (Radnoti, California, USA) with $15 \mathrm{~mL}$ of Krebs solution at $37^{\circ} \mathrm{C}$, continuously bubbled with $95 \% \mathrm{O}_{2}$ and $5 \% \mathrm{CO}_{2}$. The contractile response of muscle strip was continuously recorded using isometric force transducers (No. TRI202PAD, Panlab, Cornellã, Spain) coupled to a computer equipped with the PowerLab 8/30 System and the Labchart data analysis software (AD Instruments, Spechbach, Germany). Strips were stretched with a preload of $1 \mathrm{~g}$, which was maintained during an equilibration period of 60 minutes. Then, strips were subjected to electrical field stimulation (EFS) using a STG 4008 MCS electrical stimulator (Multi Channel Systems, Reutlingen, Germany). EFS parameters were as follows: train duration, 10 seconds; pulse frequency, $20 \mathrm{~Hz}$; pulse duration, $400 \mu \mathrm{s}$; pulse amplitude $11 \mathrm{~V}$. This procedure was repeated 3 times spaced 4 minutes. Then the following drugs were added in the baths and after a 10 minutes incubation period the same EFS stimulation protocol was repeated. The following drugs were added sequentially, nitro-L-arginine methyl ester (L-Name, $50 \mu \mathrm{M}$; Sigma-Aldrich, Merck, Darmstadt, Germany), an inhibitor of the nitric oxide synthase (NOS); and then atropine ( $1 \mu \mathrm{M}$; Sigma-Aldrich, Merck, Darmstadt, Germany), a muscarinic receptor antagonist. Next, tissues were washed 5 times over a 10 min period and was then allowed to recover for an additional $20 \mathrm{~min}$. At the end of each experiment, using bethanechol $\left(10^{-9}-10^{-2} \mathrm{M}\right)$, a selective muscarinic receptor agonist (Millipore Sigma, Burlington, MA, USA), a doseresponse curve was created by measuring the area under the curve of the bethanechol-induced contraction for 2 min after bethanechol was added. All values were normalized to the tissue weight. 


\section{Blood sampling and corticosterone assessment}

Mice were bled from their tail, at $\mathrm{J} 0$ and at $\mathrm{J} 4$, at the end of the $1 \mathrm{~h}$ WAS test (as described in figure 1). Samples were obtained by quickly clipping the distal tip of the tail with a razor blade and collecting $50 \mu \mathrm{L}$ of blood into EDTA containing tubes.

In order to minimize hormonal variability due to circadian fluctuations, all procedures were performed during circadian cycle of the diurnal corticosterone rhythm, before 11:00 AM. After collection, blood samples were centrifuged at $3200 \mathrm{tr} / \mathrm{min}$, for $15 \mathrm{~min}$ at $4^{\circ} \mathrm{C}$ and then plasma samples were kept at $-20^{\circ} \mathrm{C}$. Corticosterone plasma levels were measured by enzymatic immunoassay according to the manufacturer's instructions (Laboratoire IDS, Pouilly en Auxois, France).

\section{Acetylcholine assay}

After mice killing, portions of the distal colon were removed and lysed in RIPA buffer (Merck Millipore, Fontenay-sous-Bois, France) containing phosphatase inhibitor cocktail III (Sigma-Aldrich, Merck, Darmstadt, Germany) and protease inhibitors cocktail (Roche, Neuilly-sur-Seine, France). Briefly, tissues were crushed in the RIPA buffer using a "Precellys 24" tissue homogenizer (Bertin technologies, St Quentin-en-Yvelines, France), followed by sonication with "vibracell 75 186" device (Sonics, Newton CT, USA). The amount of proteins was assessed with Bradford reagent using a BioPhotometer D30 spectrophotometer (Eppendorf, Montesson, France). Acetylcholine (ACh) concentration was determined in tissue homogenates using Amplex Red, acetylcholine/ acetylcholinesterase assay kit (Invitrogen Thermofisher Scientific, Waltham, MA USA) and normalized with protein amount.

\section{Western Blot analysis}

As previously described (30), proteic extraction from colonic tissues was performed with NucleoSpin RNA/Protein Kit (Macherey-Nagel, Hoerdt, France, Cat\# 740966) according to the manufacturer instructions. Samples were further prepared for electrophoresis by diluting with NuPAGE sample buffer (Life Technologies, Saint-Aubin, France, Cat\# NP0008) then heated at $98^{\circ} \mathrm{C}$ for $5 \mathrm{~min}$. Lysates were separated using the NuPAGE 4-12\% Bis-Tris gels (Life Technologies, Cat\# NP0336BOX) together with the 2-(N-morpholino)ethanesulfonic acid/sodium dodecyl sulfate running buffer (Life Technologies, Cat\# IB23002) before electrophoretic transfer to nitrocellulose membranes (Life Technologies, Cat\# NP0002) with the iBlot2 Dry Blotting System (Life Technologies, Cat\# IB21001). Membranes were then blocked for $1 \mathrm{~h}$ at $21^{\circ} \mathrm{C}$ in Tris-buffered saline (Sigma, Cat\# T5912) with 0.1\% (v/v) Tween-20 (Sigma, Cat\# P1379) and $5 \%(\mathrm{w} / \mathrm{v})$ non-fat dry milk and incubated overnight at $4{ }^{\circ} \mathrm{C}$ with the following primary antibodies: rabbit anti-GR (D8H2, 3660S 1:500, Cell Signaling, Danvers, Massachusetts, USA), mouse monoclonal anti- $\beta$-actin (1 : 10000; Sigma, Cat\#A5441, RRID:AB_476744). Bound antibodies were detected with horseradish peroxidase-conjugated anti-rabbit (Life Technologies Cat\# 31460, diluted $1: 5000$ ) or antimouse antibodies (Sigma, Cat\# A9044, diluted $1: 5000$ ) and visualized by enhanced chemiluminescent detection (Biorad, Clarity ECL, Marnes-la-Coquette, France, Cat\# 170-5061).

\section{Quantitative PCR analysis}


As previously described (31), one $\mu \mathrm{g}$ purified mRNA by Nucleospin RNA/Protein kit was denatured and was converted to cDNA using the SuperScript III Reverse Transcriptase (Life Technologies). qPCR was performed using a StepOnePlus RealTime PCR Instrument (Life Technologies) with a FastSYBR Green Master Mix kit (Applied Biosystems, Foster City, CA, USA). Ribosomal protein S6 (RPS6) transcript was used as a reference. The relative expression of the gene of interest was measured by the $2-\Delta \Delta \mathrm{Ct}$ method. The following primers were used:

- NR3C1 \# NM_008173, forward: 5'-GCAGTGGAAGGACAGCACAAT-3' ; reverse: 5'CGTTTTTCGAGCTTCCAGGTTC-3'

- RPS6 forward: 5'-GAAGCGCAAGTCTGTTCGTG-3' ; Reverse : 5'- GTCCTGGGCTTCTTACCTTCT-3'

\section{Immunofluorescence staining}

Distal colon segments were fixed in 0.1M PBS containing 4\% paraformaldehyde at room temperature for $3 \mathrm{~h}$. Whole mounts of longitudinal muscle and myenteric plexus were obtained by microdissection and were permeabilized with PBS containing $10 \%$ horse serum (HS) and $4 \%$ Triton X-100 for $2 \mathrm{~h}$ at room temperature. Tissues were then incubated with the following primary antibodies: rabbit anti-ChAT (1:1000, a gift from Professor M. Schemann, Hannover, Germany (32), mouse anti-neuronal NOS (nNOS; 610308, 1:500; BD Biosciences), rabbit anti-GR (D8H2, 3660 S 1:500, Cell Signaling), human anti-Hu (gift from the CHU of Nantes; 1:5000) diluted in PBS containing 10\% horse serum and 0,5\% Triton X-100 for 24 or $48 \mathrm{~h}$ at room temperature. After washing, tissues were incubated for $2 \mathrm{~h}$ at room temperature with the appropriate secondary antibodies, respectively, anti-rabbit CY5 (1:500), anti-rabbit CY3 (1:500) and antihuman FP488 (1:200), and mounted Glycerol 60\% (vol/vol) (Thermo Fisher Scientific). Nuclei were stained with 4' 6-Diamidino-2-phenylindole dihydrochloride (DAPI D9542; 1:10000; Sigma Aldrich, Paris, France).

\section{Image analysis and quantification}

Images from immunostained tissues were acquired with a digital camera (Axiozoom, Carl Zeiss, United States). The number of Hu-, ChAT-, nNOS- and GR-neurons was counted in 20 ganglia per animal. The data were expressed as the percentage of ChAT-, nNOS- or GR-neurons normalized to the total number of Hu-neurons.

For GR immunostaining, confocal microscopy was performed using Nikon A1R confocal inverted microscope (Nikon France SAS, Champigny sur Marne, France) with a Nikon X60 Plan-Apo numerical aperture (NA) 1.4 oil-immersion objective (MicroPICell core facility).

Among these 20 ganglia, 10 were used to measure the intensity of cytoplasmic and nuclear expression of GR receptor. The DAPI-GR co-labelling was used to determine the intensity of nuclear expression of the GR receptor within each neuron of a ganglion, as the Hu-GR co-labelling was used to determine the total intensity of the GR receptor expression within each neuron of a ganglia. The intensity of GR cytoplasmic 
expression could then be deduced. The measurement of the nucleo-cytoplasmic ratio of GR expression was determined.

\section{Statistical analyses}

Statistical analyses were performed using GraphPad Prism 5 (GraphPad Software, San Diego, CA, USA). Data are represented as means +/- SD. Group comparisons were made using the Mann-Whitney U test or by 2-way ANOVA and Dunnett's multiple comparisons test as indicated. Values of $p<0.05$ were considered statistically significant.

\section{Results}

\section{Enteric neurons of mice distal colonic ENS express the GR glucocorticoid receptor}

We first aimed to determine whether enteric neurons express GR receptors. We therefore performed GR immunohistochemical labeling in whole mounts of longitudinal muscle -myenteric plexus of distal colon segments. Neurons were stained using antibodies directed against neuronal protein Hu. Using high resolution confocal microscopy, we showed that GR is expressed in a large proportion of Huimmunoreactive (IR) enteric neurons (figure 2A-D). In addition, GR was preferentially located in the nucleus of Hu-IR cells as observed using the orthogonal viewing mode (figure 2E). GR expression was also reported in other non-neuronal cells within and outside enteric ganglia.

\section{Stress increases nuclear translocation of GR}

We next aimed to determine whether RASt modulates GR expression in enteric neurons. We first showed that repeated acute WAS significantly increased serum corticosterone levels as compared to control mice (282.6 $\pm 113.9 \mathrm{ng} / \mathrm{ml}$ vs 51.8 \pm 19.2 , respectively; $\mathrm{p}<0.0001)$ (Supplemental figure S1). Next, we showed that, in Hu-IR cells, the intensity of GR expression was not modified by RASt (data not shown). Furthermore, western blot and PCR analysis of colonic tissue showed that RASt did not modify protein and mRNA expression of GR as compared to control (Supplemental figure S2). However, using immunohistochemistry we were able to show that the ratio of the intensity of nuclear GR expression over cytoplasmic GR expression was significantly increased by RASt as compared to control (figure 3).

\section{RASt-induced phenotypic modulation of ENS neurons is mediated by $\mathrm{GR}$ in mice distal colon}

We next aimed to determine whether RASt modulates the expression of two enzymes regulating the synthesis of two key neuromediators involved in regulation of gut motility, ie choline acetyltransferase (ChAT) and nitric oxide synthase (NOS) (figure 4A). We first showed that repeated acute WAS did not modify the number of neurons (identified by $\mathrm{Hu}$ ) as compared to control (figure 4B). Interestingly, repeated acute WAS induced a significant $63 \%$ increase in the proportion of ChAT-IR neurons as compared to control (figure 4C). However, RASt did not modify the proportion of nNOS-IR neurons (figure 
4D). We next showed that the increase in the proportion of ChAT-IR neurons was associated with a significant increase in ACh concentration in colonic tissue as compared to control (figure 4E). Interestingly, RASt-induced increase in ACh colonic tissue level was prevented by pretreatment of mice with CORT108297 (85mg/kg), the selective GR receptor antagonist, prior RASt.

\section{RASt increases colonic motility in a GR dependent manner}

Thereafter, we aimed to determine whether RASt-induced changes in ENS phenotype were associated with changes in colonic motor functions in a GR dependent manner.

First, we analyzed, ex vivo in organ chamber, the neuromuscular response to electrical field stimulation (EFS) of segments of distal colon. EFS-induced contractile responses were analyzed in the absence or presence of L-NAME and/or atropine (figure $5 A$ ). The EFS-induced AUC was larger in WAS mice as compared to controls $(n=16 ; p<0.001)$ (figure $5 \mathrm{~B})$. In the presence of L-NAME, EFS-induced AUC was still significantly larger in WAS as compared to controls $(n=16 ; p=0.026)$ (figure $5 \mathrm{~B}$ ). Finally, in the presence of atropine, EFS-induced AUC were significantly reduced in both WAS and control mice $(n=16 ; p$ $=0.56$ ) (figure 5B). However, the amplitude of the atropine-sensitive AUC component was higher in WAS group as compared to controls ( $n=16 ; p=0.001$ ) (figure $5 \mathrm{C}$ ). In order to exclude that differential response to atropine between WAS and control is reflecting a differential sensitivity of cholinergic receptor on muscle, we measured the muscle response to cholinergic muscarinic agonist (betanechol) stimulation. We showed that bethanechol induced a similar dose-dependent increase in contractile response in tissues of both control and WAS mice (figures 5D and E).

Finally, we aimed to determine whether changes in neuromuscular transmission induced by WAS in the distal colon were associated with functional changes in vivo. We showed that FPO were significantly higher in WAS mice compared to control, with respectively $5.8 \pm 4.3$ in control mice versus $12.3 \pm 3.5$ in WAS mice $(p<0.001 ; n=X)$ (figure 5F). Interestingly, we showed that pretreatment of WAS mice with CORT108297 (85mg/kg) prevented WAS increase in FPO (figure 5G-H). Basal administration of CORT108297 did not modify FPO.

\section{Discussion}

Our study demonstrates for the first time that myenteric neurons express the glucocorticoid receptor GR in the distal colon. We further showed that RASt enhanced nuclear translocation of GR in myenteric neurons, induced an increase in ChAT-IR neurons, acetylcholine tissue level in distal colon, and enhanced cholinergic neuromuscular transmission. We also showed that RASt induced increase in motility and in distal colonic acetylcholine expression were both prevented by the GR specific antagonist CORT108297. Altogether, our study suggests that RASt-induced functional changes in colonic motility are, at least partly, due to GR dependent cholinergic ENS remodeling. Our findings could therefore open novel therapeutic perspectives, by targeting GR, in gut functional disorders associated with altered HPA axis. 
The first finding of our study is the identification of GR expression by myenteric neurons in the mice colon, which represents a putative novel actor of RASt-induced effects upon gut functions. Indeed, until recently, RASt-induced increase in $\mathrm{CRH}$ has been thought to mediate the majority of stress induced effects upon gut functions $(15,33,34)$ in part via its activation of $\mathrm{CRH}$ receptors expressed by enteric neurons. However, increasing evidences have shown that corticosterone, another major stress hormone, can directly modulate neuronal activity in enteric neurons. First, $11-\beta$ hydroxy steroid deshydrogenase

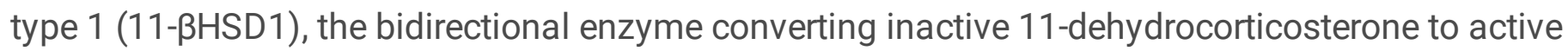
corticosterone, has been identified in populations of enteric neurons (24). 11- $\beta H S D 1$ is largely expressed in liver, adipose tissue and in brain tissue (20). While hepatic 11-BHSD1 activity appears to maintain circulating GC concentrations, 11- $\beta$ HSD1 activity in hippocampal and hypothalamic neurons appears to limit GC concentrations by activating the negative HPA feedback (35). In the ENS, the role of 11- $\beta$ HSD1 remains however largely unknown. Furthermore, acute corticosterone application at a high concentration was shown to increase intracellular calcium in myenteric neurons while long term (20h) incubation of ENS culture with corticosterone at concentrations similar to those observed during stress, reduced EFS induced $\mathrm{Ca}_{2}{ }^{+}$response in neurons (24). Finally, addition of corticosterone to primary culture of ENS was reported to increase the proportion of ChAT-IR neurons (23). Interestingly, increased enteric cholinergic activity was shown to be induce in a porcine model of early weaning stress that represent a psychological model of HPA axis activation (36). Furthermore, besides regulation of neuromediator expression, corticosterone also increased expression of synaptic associated protein such as synaptobrevin (24) in the ileum. However, the role of GR in mediating corticosterone effects upon ENS and GI functions remained elusive.

Expression of GR in the gut has been previously reported to occur in various cells of the GI tract, i.e. epithelial and immune cells of the lamina propria $(37,38)$. Our study is the first of our knowledge to demonstrate expression of GR in enteric neurons. Expression of GR has been previously described in other neuronal cell types of the peripheral nerve system such as neurons of the dorsal root ganglia and also in region-specific part of the brain (21). The GC/GR interactions in central neurons is associated with morphological plasticity and alterations of neuronal functions (39). However, these GR-induced neuronal modifications vary with stress and cell type (40). At the central level, it has been reported that the effects of acute exposure to glucocorticoids were rather associated with increased synaptic plasticity while more chronic exposure is associated with dendritic atrophy $(39,40)$. Whether region specific expression of GR and/or cell type specific expression of GR also exist in the ENS remains currently unknown. Here we demonstrate that GR is expressed by Hu-IR cells, enteric neurons, but also by non-Hu-IR cells. The nature of these non-Hu cells in myenteric plexus remain also to be characterized yet.

RASt increased the ratio of nuclear/cytoplasmic localization of GR in enteric neurons. This finding is consistent with the nuclear translocation of GR upon its binding with its ligand, leading to activation of GR dependent transcriptomic response (41). Similar findings are observed in other neurons of CNS, but have never been reported in the ENS. As a consequence of GR activation by RASt, our study demonstrated an increase of ChAT-IR neurons, an increased ACh tissue level and ultimately, an increased cholinergic 
neuromuscular transmission. Although, it is well known that acute stress can activate myenteric neurons such as cholinergic ones (42), no study has identified the ability of GC (or CRH) to modulate ENS phenotype, in particular its neuromediator contents. While $\mathrm{CRH}$ mediated effects can be considered as short-term response, leading to acute activation of enteric neurons $(43,44), G C / G R$ would preferentially induce long term changes in neurons as GR are involved in regulation of transcriptional activity and gene expression (45). Our results in the ENS are reminiscent to others obtained in the brain following GR activation. Indeed, although direct modulation of ChAT gene by GR complex has not been described yet, some studies in the CNS suggest that GC could indirectly activate ChAT expression by modulating activity of neurotrophic factors like nerve growth factor (46-48). Another pathway potentially involved in GR induced modulation of cholinergic phenotype is the ability of GC to downregulate NF-KB expression in neuron (36). Since NF-KB acts as a repressor of ChAT transcription, GR induce NF-kB inhibition would ultimately lead to increase ChAT expression (49). Furthermore, the impact of GR activation upon regulation of ENS phenotype appeared also to be specific as the proportion of nitrergic neurons was not affected. The impact of GR activation upon other neuronal functions such as neuronal connectivity and/or survival remains also to be determined in the future.

Finally, our study demonstrated the efficacy of a GR specific antagonist at preventing neuromediator and functional remodeling induced by RASt. Indeed, pretreatment with CORT108297 decreased FPO and ACh tissue level in mice subjected to WAS. CORT108297 is a GR specific antagonist with a strong affinity (26) and with the advantage, as compared to the other GR antagonist RU486, to be devoid of progesterone receptor inhibitory effect (50). CORT108297 has been shown to potently suppress corticosterone responses to forced swim test (FST) and restraint stress (51). Whether CORT108297 has local action or not within the gut remains to be further explored. Even if molecular effect of CORT108297 remains to be elucidated, our data suggest the use of a specific GR antagonist such as CORT108297 as a novel pharmacological tool in the management of stress-related digestive pathologies such as irritable bowel syndrome (IBS) (52) or even for digestive disorders in the population with chronic adrenal insufficiency (53). In particular, in IBS the use of CRF-R1 antagonists to improve digestive is inconclusive to date (54); therefore targeting GR with CORT108297 could represent a promising therapeutic new avenue in these patients.

\section{Abbreviations}

ACh

Acetylcholine

ChAT

Choline Acetyltransferase

CNS

central nervous system

EFS

Electrical field stimulation

ENS 
Enteric Nervous System

GC

Glucocorticoids

GI

Gastro intestinal

GR

Glucocorticoid receptor

HPA

Hypothalamic-pituitary-adrenal

nNOS

neuronal Nitric oxide synthase

WAS

Water avoidance stress

RASt

Repeated acute stress

\section{Declarations}

\section{Acknowledgments}

The authors are grateful to Dr Hazel Hunt (Corcept) who kindly provided CORT208297 and to AnneSophie Ducoq and Camille Renaud for technical assistance. We acknowledge the IBISA MicroPICell facility (Biogenouest), member of the national infrastructure France-Bioimaging supported by the French national research agency (ANR-10-INBS-04)

\section{Author contributions (names must be given as initials)}

MN and KBN conceived and designed the study. JB, CG, PA, TO, TD conceived and conducted the experiment(s). PN performed immunohistochemical analysis. $M N, K B N, J B$ and $C G$ analyzed the results. $\mathrm{JB}, \mathrm{KBN}, \mathrm{PN}, \mathrm{MN}$ wrote the MS. DM,PA,TD critically reviewed the manuscript. All authors reviewed the manuscript.

\section{Additional Information (including a Competing Interests Statement)}

The authors declare no competing interests.

This work was funded in part by the French non-profit patient association "Adrenals" (http://www.surrenales.com).

\section{References}


1. Meerveld BG-V, Johnson AC. Mechanisms of Stress-induced Visceral Pain. J Neurogastroenterol Motil. 30 janv 2018;24(1):7-18.

2. Bhatia V, Tandon RK. Stress and the gastrointestinal tract. J Gastroenterol Hepatol. mars 2005;20(3):332-9.

3. Reed DE, Zhang Y, Beyak MJ, Lourenssen S, Blennerhassett MG, Paterson WG, et al. Stress increases descending inhibition in mouse and human colon. Neurogastroenterol Motil. avr 2016;28(4):569-80.

4. Bonaz B, Baciu M, Papillon E, Bost R, Gueddah N, Le Bas JF, et al. Central processing of rectal pain in patients with irritable bowel syndrome: an fMRI study. Am J Gastroenterol. mars 2002;97(3):654-61.

5. Gourcerol G, Wu SV, Yuan P, Pham H, Miampamba M, Larauche M, et al. Activation of CorticotropinReleasing Factor Receptor 2 Mediates the Colonic Motor Coping Response to Acute Stress in Rodents. Gastroenterology. 1 mai 2011;140(5):1586-1596.e6.

6. Taché Y. Corticotrophin-releasing factor 1 activation in the central amygdale and visceral hyperalgesia. Neurogastroenterol Motil. janv 2015;27(1):1-6.

7. Larauche M, Gourcerol G, Wang L, Pambukchian K, Brunnhuber S, Adelson DW, et al. Cortagine, a CRF1 agonist, induces stresslike alterations of colonic function and visceral hypersensitivity in rodents primarily through peripheral pathways. Am J Physiol Gastrointest Liver Physiol. juill 2009;297(1):G215-227.

8. Taché Y, Kiank C, Stengel A. A role for corticotropin-releasing factor in functional gastrointestinal disorders. Curr Gastroenterol Rep. août 2009;11(4):270-7.

9. Martínez V, Wang L, Rivier J, Grigoriadis D, Taché Y. Central CRF, urocortins and stress increase colonic transit via CRF1 receptors while activation of CRF2 receptors delays gastric transit in mice. $J$ Physiol. 1 avr 2004;556(Pt 1):221-34.

10. Tsukamoto K, Nakade Y, Mantyh C, Ludwig K, Pappas TN, Takahashi T. Peripherally administered CRF stimulates colonic motility via central CRF receptors and vagal pathways in conscious rats. Am J Physiol Regul Integr Comp Physiol. juin 2006;290(6):R1537-1541.

11. Taché Y, Bonaz B. Corticotropin-releasing factor receptors and stress-related alterations of gut motor function. J Clin Invest. janv 2007;117(1):33-40.

12. Neunlist M, Van Landeghem L, Mahé MM, Derkinderen P, des Varannes SB, Rolli-Derkinderen $M$. The digestive neuronal-glial-epithelial unit: a new actor in gut health and disease. Nat Rev Gastroenterol Hepatol. févr 2013;10(2):90-100.

13. Furness JB, Callaghan BP, Rivera LR, Cho H-J. The enteric nervous system and gastrointestinal innervation: integrated local and central control. Adv Exp Med Biol. 2014;817:39-71.

14. Spencer NJ, Hu H. Enteric nervous system: sensory transduction, neural circuits and gastrointestinal motility. Nat Rev Gastroenterol Hepatol. juin 2020;17(6):338-51.

15. Rivier CL, Grigoriadis DE, Rivier JE. Role of corticotropin-releasing factor receptors type 1 and 2 in modulating the rat adrenocorticotropin response to stressors. Endocrinology. juin 2003;144(6):2396403. 
16. Liu S, Karo A, Agterberg S, Hua H, Bhargava A. Effects of stress-related peptides on chloride secretion in the mouse proximal colon. Neurogastroenterology \& Motility. 2021;33(4):e14021.

17. Nicole A. Datson. Central corticosteroid actions: Search for gene targets. European Journal of Pharmacology. 30 janv 2008;

18. Oakley RH, Cidlowski JA. The biology of the glucocorticoid receptor: New signaling mechanisms in health and disease. Journal of Allergy and Clinical Immunology. 1 nov 2013;132(5):1033-44.

19. Revollo JR, Cidlowski JA. Mechanisms generating diversity in glucocorticoid receptor signaling. Ann N Y Acad Sci. oct 2009;1179:167-78.

20. Chapman K, Holmes M, Seckl J. 11ß-Hydroxysteroid Dehydrogenases: Intracellular Gate-Keepers of Tissue Glucocorticoid Action. Physiological Reviews. 1 juill 2013;93(3):1139-206.

21. Lerch JK, Alexander JK, Madalena KM, Motti D, Quach T, Dhamija A, et al. Stress Increases Peripheral Axon Growth and Regeneration through Glucocorticoid Receptor-Dependent Transcriptional Programs. eNeuro. août 2017;4(4).

22. Buurstede JC, Weert LTCM van, Colucci P, Gentenaar M, Viho EMG, Koorneef LL, et al. Hippocampal glucocorticoid target genes associated with enhancement of memory consolidation. European Journal of Neuroscience [Internet]. [cité 4 août 2021];n/a(n/a). Disponible sur: https://onlinelibrary.wiley.com/doi/abs/10.1111/ejn.15226

23. Aubert P, Oleynikova E, Rizvi H, Ndjim M, Le Berre-Scoul C, Grohard PA, et al. Maternal protein restriction induces gastrointestinal dysfunction and enteric nervous system remodeling in rat offspring. FASEB J. janv 2019;33(1):770-81.

24. Lowette $K$, Tack J, Vanden Berghe P. Role of corticosterone in the murine enteric nervous system during fasting. American Journal of Physiology-Gastrointestinal and Liver Physiology. 1 nov 2014;307(9):G905-13.

25. Jain P, Hassan AM, Koyani CN, Mayerhofer R, Reichmann F, Farzi A, et al. Behavioral and molecular processing of visceral pain in the brain of mice: impact of colitis and psychological stress. Front Behav Neurosci. 2015;9:177.

26. Meyer M, Gonzalez Deniselle MC, Hunt H, de Kloet ER, De Nicola AF. The selective glucocorticoid receptor modulator CORT108297 restores faulty hippocampal parameters in Wobbler and corticosterone-treated mice. J Steroid Biochem Mol Biol. sept 2014;143:40-8.

27. Rincel M, Aubert P, Chevalier J, Grohard P-A, Basso L, Monchaux de Oliveira C, et al. Multi-hit early life adversity affects gut microbiota, brain and behavior in a sex-dependent manner. Brain Behav Immun. août 2019;80:179-92.

28. Tasselli M, Chaumette T, Paillusson S, Monnet Y, Lafoux A, Huchet-Cadiou C, et al. Effects of oral administration of rotenone on gastrointestinal functions in mice. Neurogastroenterol Motil. mars 2013;25(3):e183-193.

29. Aubert P, Guinobert I, Blondeau C, Bardot V, Ripoche I, Chalard P, et al. Basal and Spasmolytic Effects of a Hydroethanolic Leaf Extract of Melissa officinalis L. on Intestinal Motility: An Ex Vivo Study. J Med Food. juill 2019;22(7):653-62. 
30. Prigent A, Gonzales J, Durand T, Le Berre-Scoul C, Rolli-Derkinderen M, Neunlist M, et al. Acute inflammation down-regulates alpha-synuclein expression in enteric neurons. J Neurochem. mars 2019;148(6):746-60.

31. Bodin R, Paillé V, Oullier T, Durand T, Aubert P, Le Berre-Scoul C, et al. The ephrin receptor EphB2 regulates the connectivity and activity of enteric neurons. J Biol Chem. nov 2021;297(5):101300.

32. Schemann M, Sann H, Schaaf C, Mäder M. Identification of cholinergic neurons in enteric nervous system by antibodies against choline acetyltransferase. Am J Physiol. nov 1993;265(5 Pt 1):G10051009.

33. Fukudo S. Role of corticotropin-releasing hormone in irritable bowel syndrome and intestinal inflammation. J Gastroenterol. janv 2007;42 Suppl 17:48-51.

34. Taché Y, Million M, Nelson AG, Lamy C, Wang L. Role of corticotropin-releasing factor pathways in stress-related alterations of colonic motor function and viscerosensibility in female rodents. Gend Med. sept 2005;2(3):146-54.

35. Harris HJ, Kotelevtsev Y, Mullins JJ, Seckl JR, Holmes MC. Intracellular regeneration of glucocorticoids by 11 beta-hydroxysteroid dehydrogenase (11beta-HSD)-1 plays a key role in regulation of the hypothalamic-pituitary-adrenal axis: analysis of 11beta-HSD-1-deficient mice. Endocrinology. janv 2001;142(1):114-20.

36. Medland JE, Pohl CS, Edwards LL, Frandsen S, Bagley K, Li Y, et al. Early life adversity in piglets induces long-term upregulation of the enteric cholinergic nervous system and heightened, sexspecific secretomotor neuron responses. Neurogastroenterol Motil. sept 2016;28(9):1317-29.

37. Zong Y, Zhu S, Zhang S, Zheng G, Wiley JW, Hong S. Chronic Stress and Intestinal Permeability: Lubiprostone Regulates Glucocorticoid Receptor-mediated Changes in Colon Epithelial Tight Junction Proteins, Barrier Function and Visceral Pain in The Rodent and Human. Neurogastroenterol Motil. févr 2019;31(2):e13477.

38. Zheng G, Wu S-P, Hu Y, Smith DE, Wiley JW, Hong S. Corticosterone mediates stress-related increased intestinal permeability in a region-specific manner. Neurogastroenterol Motil. févr 2013;25(2):e127139.

39. Madalena KM, Lerch JK. Glucocorticoids and nervous system plasticity. Neural Regen Res. janv 2016;11(1):37-41.

40. Madalena KM, Lerch JK. The Effect of Glucocorticoid and Glucocorticoid Receptor Interactions on Brain, Spinal Cord, and Glial Cell Plasticity. Neural Plast. 2017;2017:8640970.

41. Meijer OC, Buurstede JC, Schaaf MJM. Corticosteroid Receptors in the Brain: Transcriptional Mechanisms for Specificity and Context-Dependent Effects. Cell Mol Neurobiol. mai 2019;39(4):53949.

42. Miampamba $M$, Million $M$, Yuan $P-Q$, Larauche $M$, Taché $Y$. Water avoidance stress activates colonic myenteric neurons in female rats. Neuroreport. 7 mai 2007;18(7):679-82.

43. Hanami M, Wood JD. Corticotropin-releasing hormone excites myenteric neurons in the guinea-pig small intestine. European Journal of Pharmacology. 28 janv 1992;211(1):23-7. 
44. Tache Y, Larauche M, Yuan P-Q, Million M. Brain and Gut CRF Signaling: Biological Actions and Role in the Gastrointestinal Tract. Curr Mol Pharmacol. 2018;11(1):51-71.

45. Petrak J, Bundzikova J, Pirnik Z, Mravec B, Kvetnansky R, Kiss A. Fos expression in tyrosine hydroxylase containing hypothalamic neurons in CRH-KO mice: effect of immobilization stress. Endocr Regul. juill 2010;44(3):89-99.

46. Shi B, Rabin SJ, Brandoli C, Mocchetti I. Dexamethasone induces hypertrophy of developing medial septum cholinergic neurons: potential role of nerve growth factor. J Neurosci. 15 nov 1998;18(22):9326-34.

47. Borges S, Coimbra B, Soares-Cunha C, Ventura-Silva AP, Pinto L, Carvalho MM, et al. Glucocorticoid programing of the mesopontine cholinergic system. Front Endocrinol (Lausanne). 2013;4:190.

48. Gonzalez SL, Saravia F, Gonzalez Deniselle MC, Lima AE, De Nicola AF. Glucocorticoid regulation of motoneuronal parameters in rats with spinal cord injury. Cell Mol Neurobiol. oct 1999;19(5):597-611.

49. Toliver-Kinsky T, Wood T, Perez-Polo JR. Nuclear factor kappaB/p49 is a negative regulatory factor in nerve growth factor-induced choline acetyltransferase promoter activity in PC12 cells. J Neurochem. déc 2000;75(6):2241-51.

50. Hunt HJ, Belanoff JK, Golding E, Gourdet B, Phillips T, Swift D, et al. 1H-Pyrazolo[3,4-g]hexahydroisoquinolines as potent $\mathrm{GR}$ antagonists with reduced $\mathrm{hERG}$ inhibition and an improved pharmacokinetic profile. Bioorg Med Chem Lett. 15 déc 2015;25(24):5720-5.

51. Solomon MB, Wulsin AC, Rice T, Wick D, Myers B, McKlveen J, et al. The selective glucocorticoid receptor antagonist CORT 108297 decreases neuroendocrine stress responses and immobility in the forced swim test. Horm Behav. avr 2014;65(4):363-71.

52. Ford AC, Lacy BE, Talley NJ. Irritable Bowel Syndrome. N Engl J Med. 29 juin 2017;376(26):2566-78.

53. Quénéhervé L, Drui D, Blin J, Péré M, Coron E, Barbara G, et al. Digestive symptoms in daily life of chronic adrenal insufficiency patients are similar to irritable bowel syndrome symptoms. Sci Rep. 13 avr 2021;11(1):8077.

54. Taché Y, Million M. Role of Corticotropin-releasing Factor Signaling in Stress-related Alterations of Colonic Motility and Hyperalgesia. J Neurogastroenterol Motil. 31 janv 2015;21(1):8-24.

\section{Figures}


a Control mice
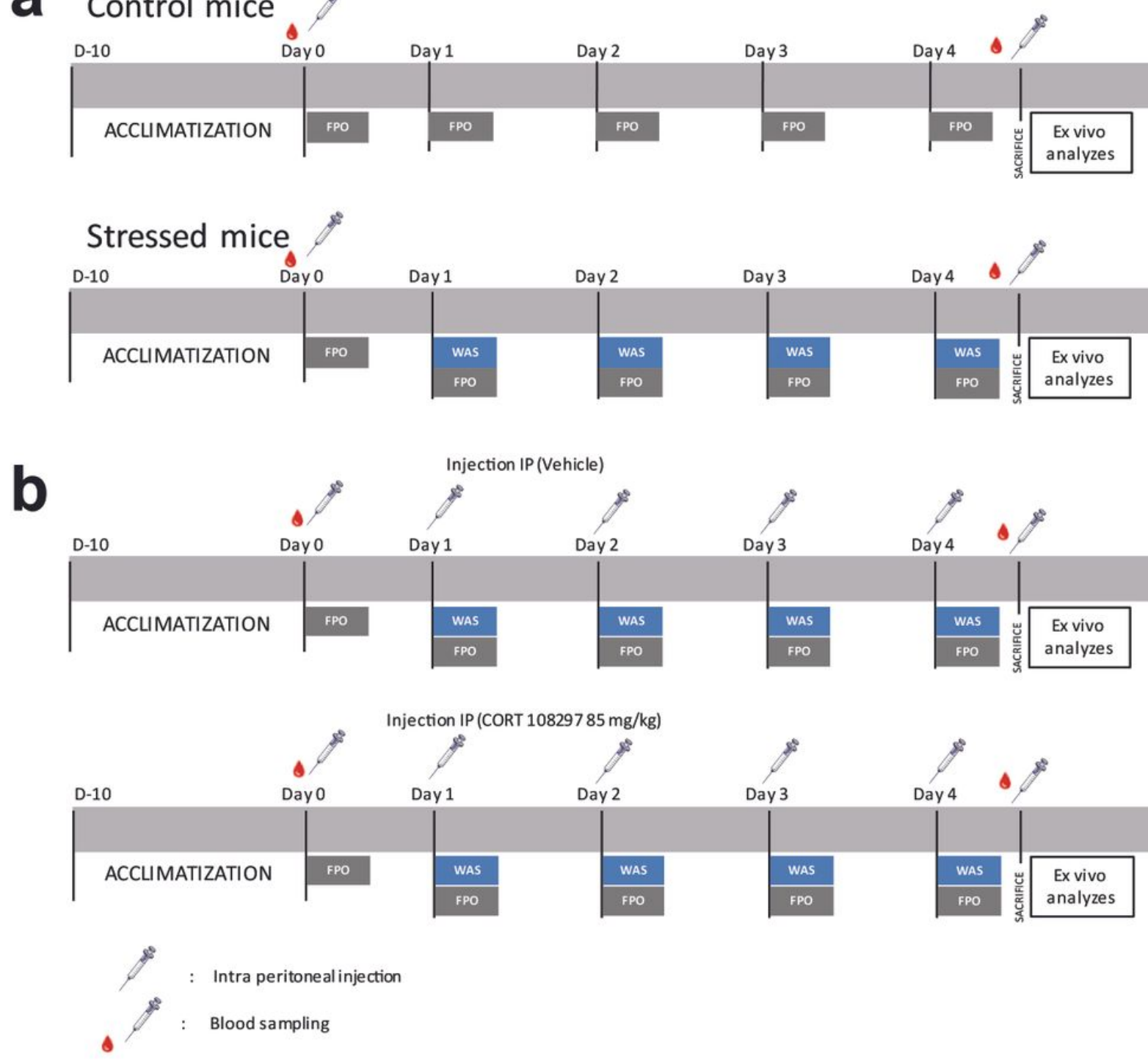

\section{Figure 1}

Experimental design (a) Schematic timeline depicting the experimental design applied to control and WAS mice for the assessment of in vivo and ex vivo colonic motility. (b) Schematic timeline depicting the experimental design applied to mice treated with CORT 108297 (85 mg/kg) or vehicle (sesame oil) for assessment of in vivo and ex vivo colonic motility. 

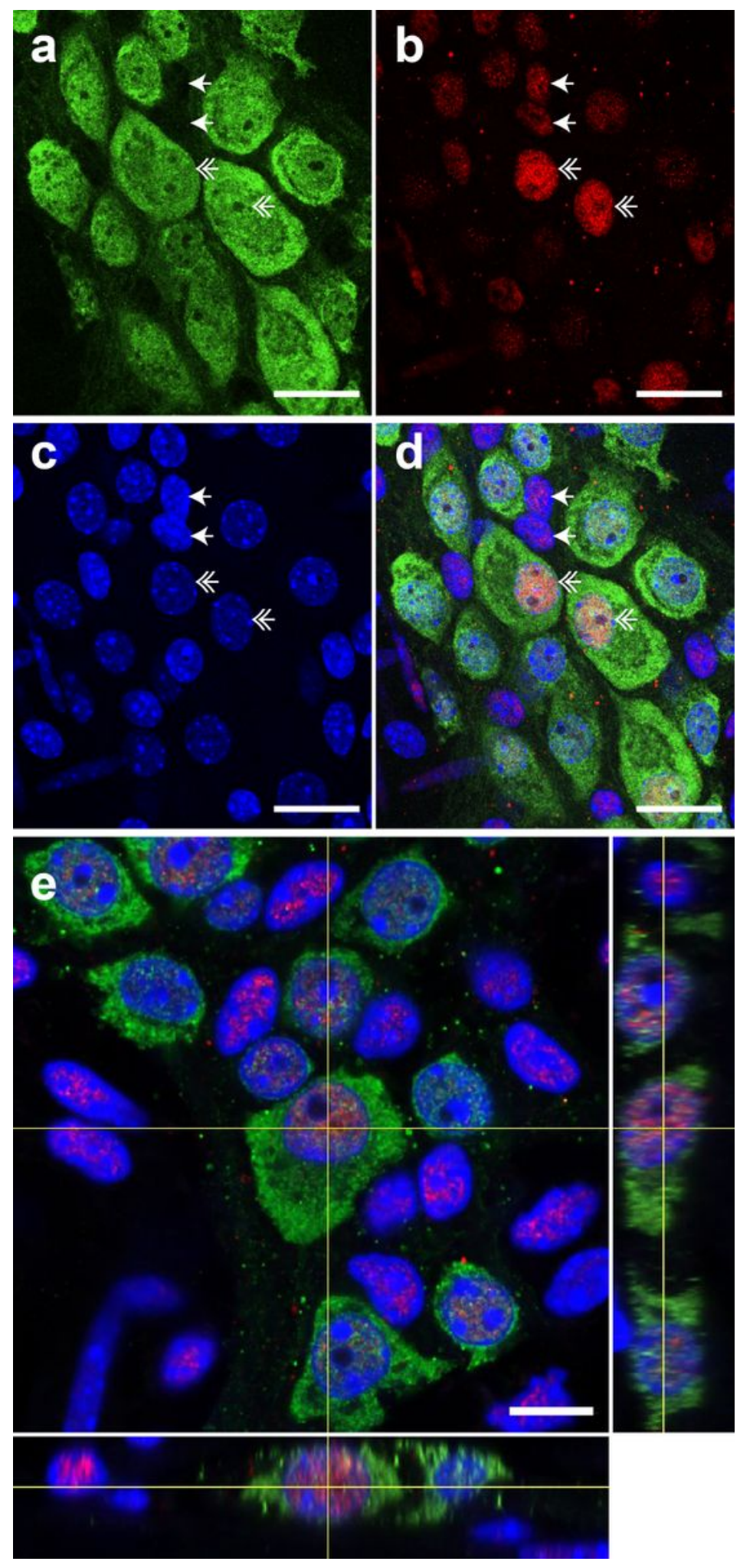

Figure 2

GR is expressed by neurons of ENS in distal colon of mice. Confocal acquisition of double immunolabeling for $\mathrm{Hu}(\mathrm{a})$ and GR (b) in the myenteric plexus of mice distal colon. 4',6-diamidino-2phenylindole (DAPI) was used for immunolabelling of nuclei (c). The merged image (d) reveals the colocalization of GR with the DAPI labeling and therefore a rather nuclear localization of GR. Scale bars of panel (a) to (d) represents $25 \mu \mathrm{m}$. Double arrows show GR expression by Hu-IR enteric neurons and 
simple arrows show GR expression by non-neuronal cells. (e) Z-reconstruction of confocal micrographs of myenteric plexus after double immunostaining with $\mathrm{Hu}$ and GR antibodies showing the preferential nuclear localization of GR in the neuron. Scale bars of image (e) represents $10 \mu \mathrm{m}$.

a
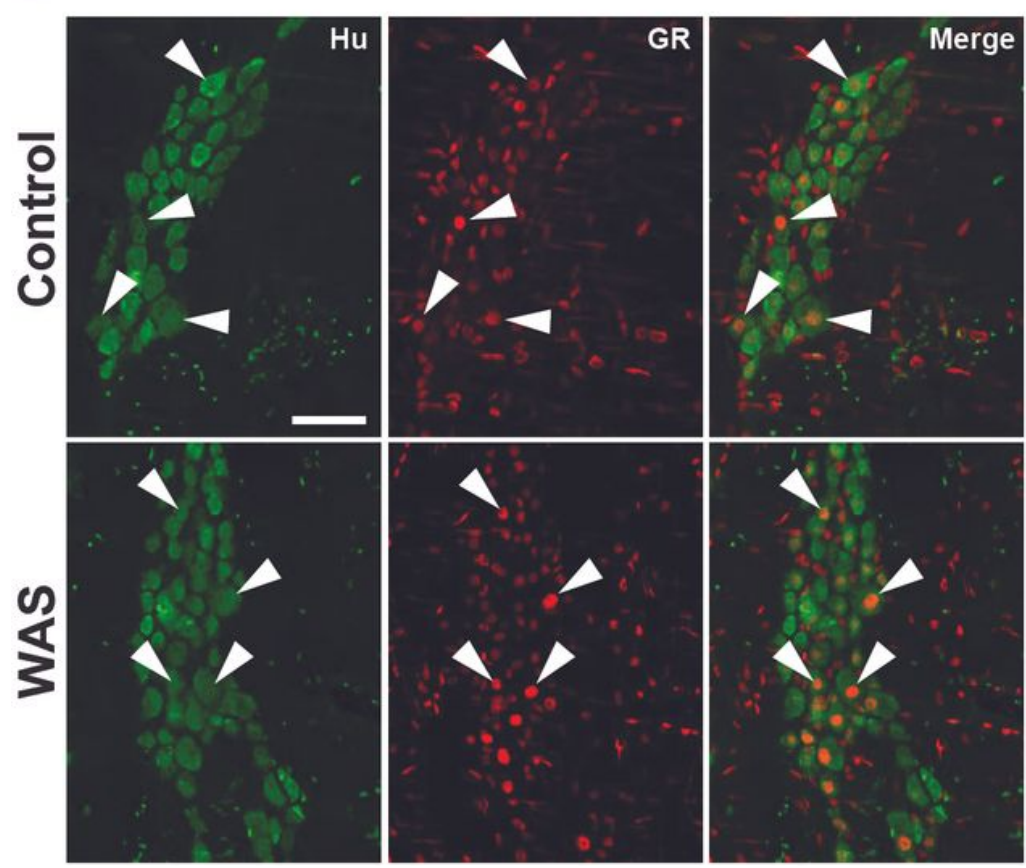

b

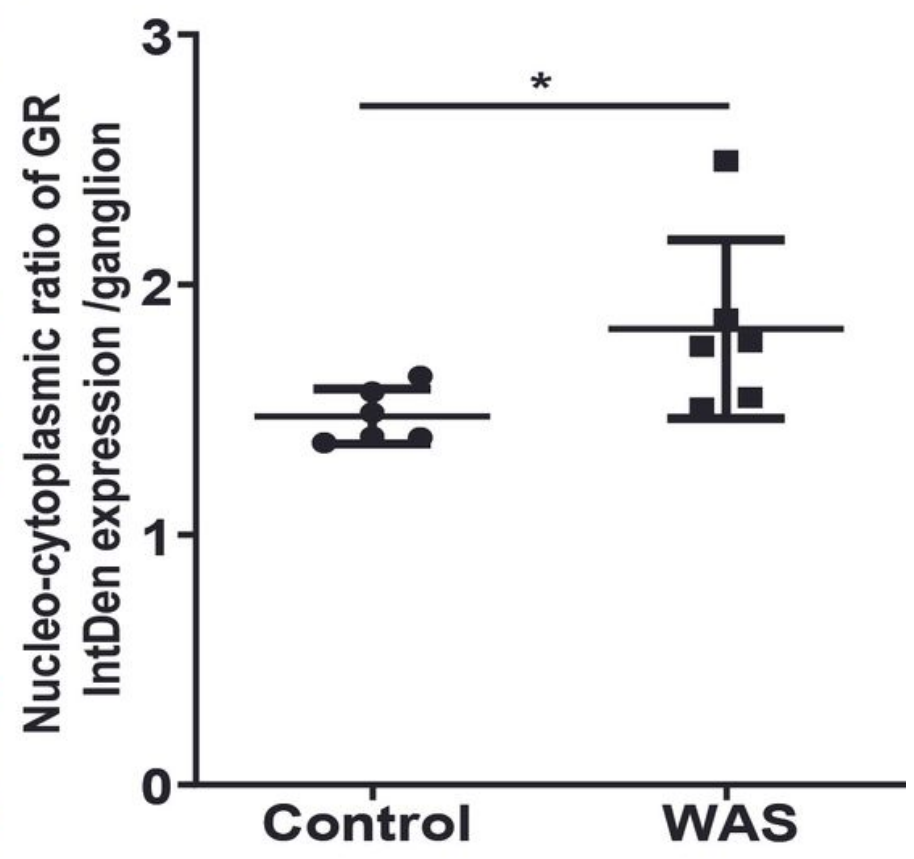

Figure 3

Stress increases the nuclear translocation of GR in neurons of the myenteric plexus (a) Double immunolabeling for Hu (green; arrowheads indicate neuron marker) and GR (red; arrows indicate glucocorticoid receptor marker in neurons) in the myenteric plexus of control and WAS mice distal colon. Scale bar represents $25 \mu \mathrm{m}$. (b) Quantitative nucleo-cytoplasmic ratio of GR labeling intensity (IntDen) per ganglion. Data are represented as means $+/-S D(n=6 /$ group) * $p<0.05$ (Mann-Whitney U test). 
a
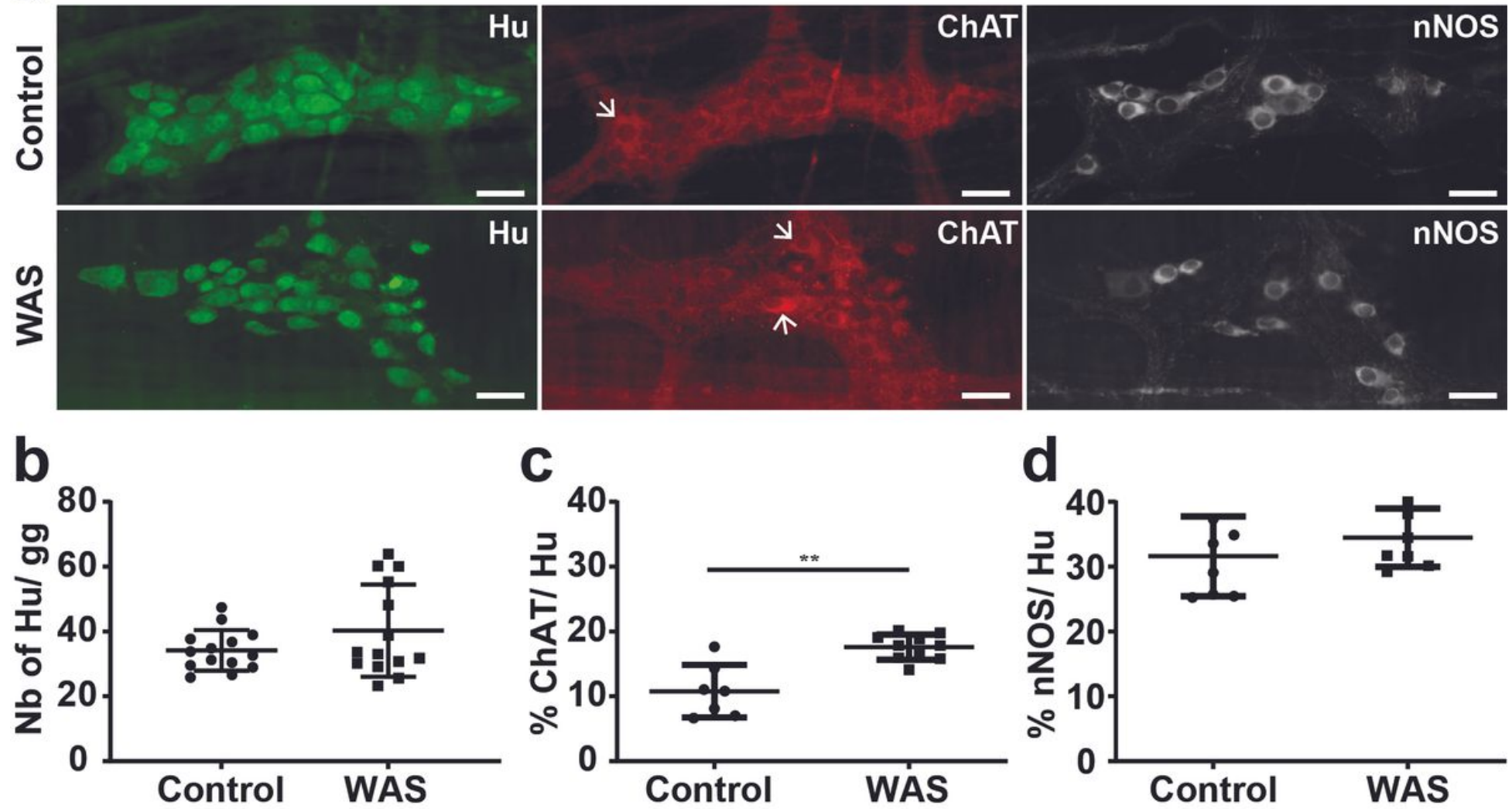

d

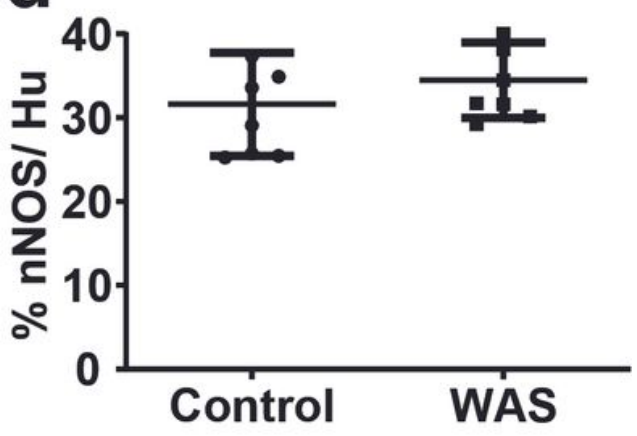

e

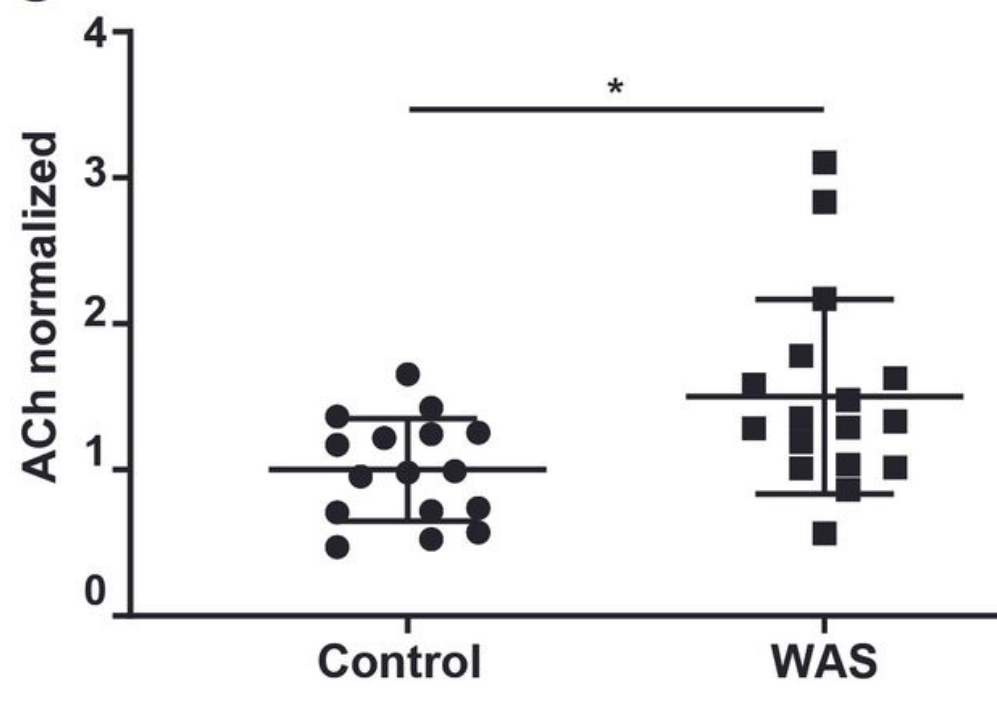

Figure 4

Stress induce modifications of enteric neuronal phenotype (a) Immunolabeling for Hu, ChAT and nNOS in the myenteric plexus of mice distal colon in control or WAS conditions (representative images are

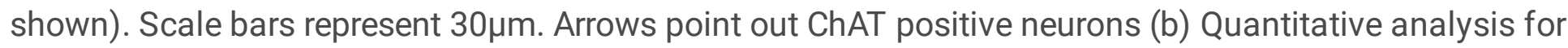
the neurons per ganglion (gg) in control and WAS groups. Data are represented as means +/- SD $(n=14 /$ group) $p=0.511$ (Mann-Whitney $U$ test) (c) Proportion of neurons expressing ChAT (ChAT/Hu) in control and WAS groups. Data are represented as means $+/-S D$ ( $n=7$ for control mice and $n=10$ for WAS mice) ${ }^{*} \mathrm{p}<0.01$ (Mann-Whitney U test) (d) Proportion of neurons expressing nNOS (nNOS/Hu) in control and 
WAS groups. Data are represented as means $+/-$ SD $(n=8 /$ group) $p=0.328$ (Mann-Whitney U test) (e) Distal colonic acetylcholine tissue levels (ACh) in control and WAS conditions +/- GR antagonist CORT108297. Data are presented as means +/- SD. One way ANOVA test and Dunnett's multiple comparisons test was used for figure (e). ${ }^{\star} p<0.05$
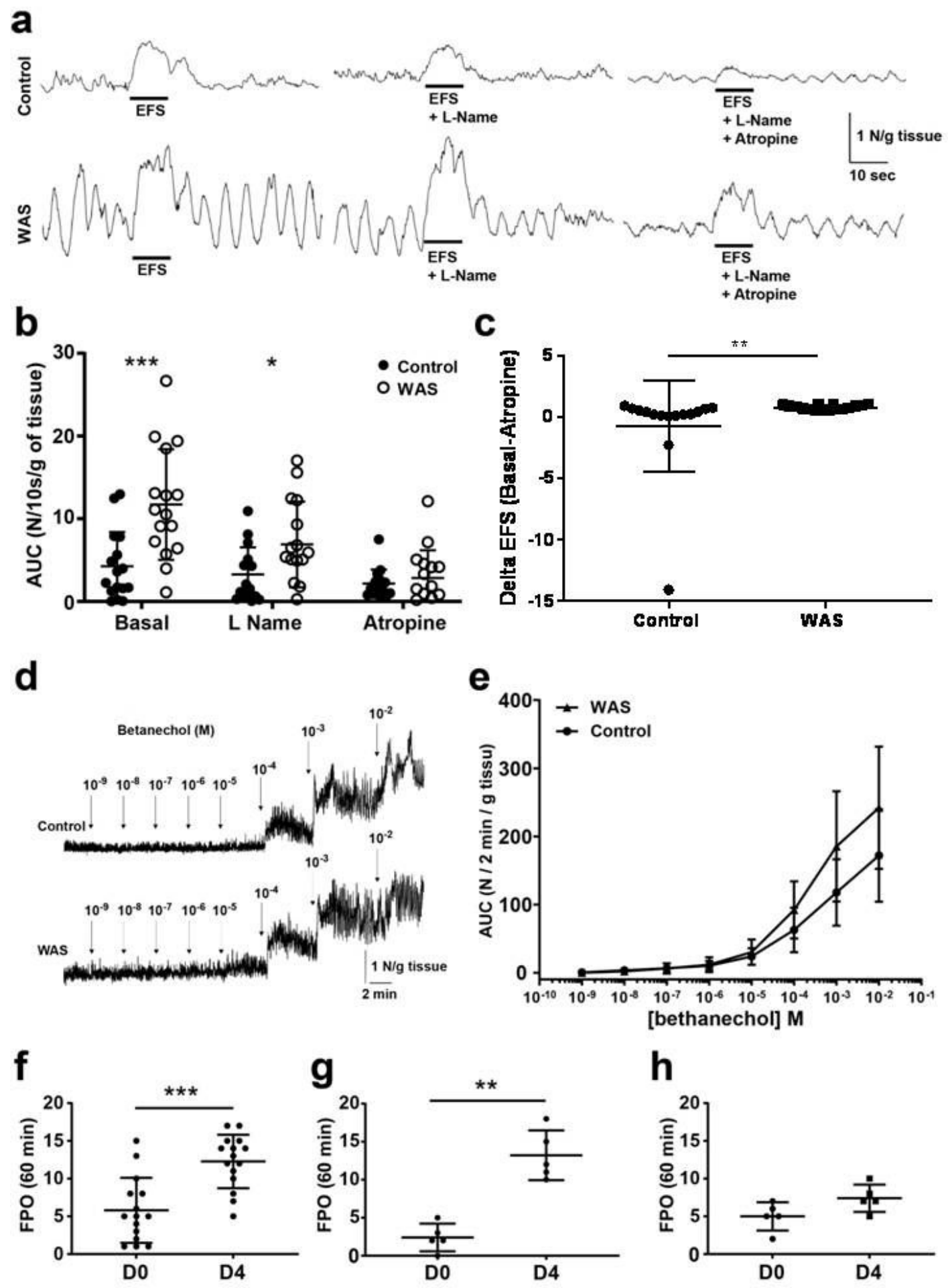


\section{Figure 5}

WAS mice exhibited increased colonic motility. Distal colonic longitudinal muscle segments were stimulated by electrical field stimulation (EFS). (a) Representative traces of EFS contractile response in basal condition, with L-Name and with L-Name + Atropine in control and WAS mice. (b) Mean EFSinduced area under curve (AUC) WAS of distal colon longitudinal muscle segment after a treatment or not with $L$ Name or Atropine ( $n=16 /$ group). (c) Variation of EFS-induced response attributed to cholinergic pathway between control and WAS mice ( $n=16 /$ group) (d) Representative traces response of isolated colon from control and WAS mice to increasing dose of bethanechol (e) Dose-response AUC contractions of distal colonic longitudinal muscular segments from control and WAS mice to increasing dose of bethanechol ( $n=16 /$ group). (f) In vivo propulsive motor function of the colon assessed by measuring FPO of mice after 1 hour of WAS treatment at day 0 before RASt and at day 4 , after 4 days of repeated WAS ( $n=16 /$ group). (g) Comparison of the in vivo propulsive motor function between mice receiving the vehicle (sesame oil), at day 0 and at day 4 ( $n=5 /$ group). (h) Comparison of the in vivo propulsive motor function between mice receiving the CORT108297, at day 0 and at day 4 ( $n=5 /$ group). Data are represented with bar as means $+/-S D * p<0.05$, ${ }^{*} p<0.01$ Mann-Whitney $U$ test were used for all figures, except figure $5 E$ were One-way ANOVA was used.

\section{Supplementary Files}

This is a list of supplementary files associated with this preprint. Click to download.

- Supplementaldata11012022.docx 